\title{
Update Breast Cancer 2021 Part 2 - Advanced Stages, Long-Term Consequences and Biomarkers
}

\author{
Update Mammakarzinom 2021 Teil 2 - \\ fortgeschrittene Stadien, Langzeitfolgen und Biomarker
}

\section{(ㄷ) (i) (오) $\ominus$}

\section{Authors}

Nina Ditsch ${ }^{1}$, Elmar Stickeler ${ }^{2}$, Annika Behrens ${ }^{3}$, Erik Belleville ${ }^{4}$, Peter A. Fasching ${ }^{3}$, Tanja N. Fehm ${ }^{5}$, Andreas D. Hartkopf $^{6}$, Christian Jackisch ${ }^{7}$, Wolfgang Janni ${ }^{8}$, Cornelia Kolberg-Liedtke ${ }^{9}$, Hans-Christian Kolberg ${ }^{10}$, Diana Lüftner $^{11}$, Michael P. Lux ${ }^{12}$, Volkmar Müller ${ }^{13}$, Andreas Schneeweiss ${ }^{14}$, Florian Schütz ${ }^{15}$, Carla E. Schulmeyer ${ }^{3}$, Hans Tesch $^{16}$, Christoph Thomssen ${ }^{17}$, Christoph Uleer ${ }^{18}$, Michael Untch ${ }^{19}$, Manfred Welslau ${ }^{20}$, Achim Wöckel ${ }^{21}$, Lena A. Wurmthaler ${ }^{3}$, Rachel Würstlein ${ }^{22}$, Marc Thill ${ }^{23}$, Bahriye Aktas ${ }^{24}$

\section{Affiliations}

1 Department of Gynecology and Obstetrics, University Hospital Augsburg, Augsburg, Germany

2 Department of Gynecology and Obstetrics, RWTH University Hospital Aachen, Aachen, Germany

3 Erlangen University Hospital, Department of Gynecology and Obstetrics, Comprehensive Cancer Center ErlangenEMN, Friedrich-Alexander University Erlangen-Nuremberg, Erlangen, Germany

4 ClinSol GmbH \& Co. KG, Würzburg, Germany

5 Department of Gynecology and Obstetrics, University Hospital Düsseldorf, Düsseldorf, Germany

6 Department of Obstetrics and Gynecology, University of Tübingen, Tübingen, Germany

7 Department of Obstetrics and Gynecology, Sana Klinikum Offenbach, Offenbach, Germany

8 Department of Gynecology and Obstetrics, Ulm University Hospital, Ulm, Germany

9 Department of Gynecology and Obstetrics, University Hospital Essen, Essen, Germany

10 Department of Gynecology and Obstetrics, Marienhospital Bottrop, Bottrop, Germany

11 Charité University Hospital, Department of Hematology, Oncology and Tumour Immunology, University Medicine Berlin, Berlin, Germany

12 Klinik für Gynäkologie und Geburtshilfe, Frauenklinik St. Louise, Paderborn, St. Josefs-Krankenhaus, Salzkotten, St. Vincenz Krankenhaus GmbH, Paderborn, Germany

13 Department of Gynecology, Hamburg-Eppendorf University Medical Center, Hamburg, Germany

14 National Center for Tumor Diseases (NCT), Heidelberg University Hospital and German Cancer Research Center, Heidelberg, Germany

15 Gynäkologie und Geburtshilfe, Diakonissen-StiftungsKrankenhaus Speyer, Speyer, Germany
16 Oncology Practice at Bethanien Hospital Frankfurt, Frankfurt, Germany

17 Department of Gynaecology, Martin-Luther-University Halle-Wittenberg, Halle (Saale), Germany

18 Praxisgemeinschaft Frauenärzte am Bahnhofsplatz, Hildesheim, Germany

19 Clinic for Gynecology and Obstetrics, Breast Cancer Center, Genecologic Oncology Center, Helios Klinikum Berlin Buch, Berlin, Germany

20 Onkologie Aschaffenburg, Aschaffenburg, Germany

21 Department of Gynecology and Obstetrics, University Hospital Würzburg, Würzburg, Germany

22 Breast Center, Department of Gynecology and Obstetrics and CCC Munich Ludwig Maximilians University, LMU University Hospital, Munich, Germany

23 Agaplesion Markus Krankenhaus, Department of Gynecology and Gynecological Oncology, Frankfurt, Germany

24 Klinik und Poliklinik für Gynäkologie, Universitätsklinikum Leipzig, Leipzig, Germany

Key words

advanced breast cancer, biomarkers, therapy, prognosis

Schlüsselwörter

fortgeschrittenes Mammakarzinom, Biomarker, Therapie,

Prognose

received

accepted after revision

23.3. 2021

published online

3.5. 2021

Bibliography

Geburtsh Frauenheilk 2021; 81: 539-548

DOI $10.1055 / a-1464-1221$

ISSN 0016-5751 


\section{(c) 2021. The Author(s).}

This is an open access article published by Thieme under the terms of the Creative Commons Attribution-NonDerivative-NonCommercial-License, permitting copying and reproduction so long as the original work is given appropriate credit. Contents may not be used for commercial purposes, or adapted, remixed, transformed or built upon. (https://creativecommons.org/licenses/by-nc-nd/4.0/)

Georg Thieme Verlag KG, Rüdigerstraße 14,

70469 Stuttgart, Germany

Correspondence

Peter A. Fasching, MD

Erlangen University Hospital, Department of Gynecology and Obstetrics, Comprehensive Cancer Center Erlangen EMN,

Friedrich Alexander University of Erlangen-Nuremberg

Universitätsstraße 21-23, 91054 Erlangen, Germany

peter.fasching@fau.de

$\oplus$

Deutsche Version unter:

https://doi.org/10.1055/a-1464-1221

\section{ABSTRACT}

This review summarises and discusses significant aspects of recently published studies on patient treatment in advanced breast cancer and on biomarkers in breast cancer. In recent years, a large number of drugs for all molecular subtypes have been developed up to phase III trials. With regard to immune checkpoint inhibitors in metastasised breast cancer, the recent discussion has centred on the best candidate for combined chemotherapy. The oral taxanes could become a new type of oral chemotherapies. There is a growing body of data on biomarkers for the use of CDK4/6 inhibitors, which could also signify further development for other molecular subtypes. New substances have been developed for metastatic HER2+ breast cancer that still result in good remission even after massive prior treatment and/or cerebral metastasis. Similarly, knowledge is growing about targeted therapies with antibody-drug conjugates (ADC) against Trop-2, which could bolster our therapeutic armoury in triple-negative breast cancer (TNBC). In addition, the clinical focus is on understanding how to maintain fertility after breast cancer treatment. Here, pooled analyses provide new insights.

\section{ZUSAMMENFASSUNG}

In dieser Übersichtsarbeit werden relevante Aspekte kürzlich veröffentlichter Studien zur Behandlung von Brustkrebspatientinnen in fortgeschrittenen Stadien sowie zu Biomarkern beim Mammakarzinom zusammenfassend dargestellt und diskutiert. In den letzten Jahren wurde eine Vielzahl von Medikamenten für alle molekularen Subtypen bis zu PhaseIII-Studien entwickelt. In Bezug auf die Immuncheckpoint-Inhibitoren wurde in letzter Zeit für die metastasierte Situation der optimale Chemotherapie-Kombinationspartner diskutiert. Mit oralen Taxanen könnte sich eine neue Art von oralen Chemotherapien etablieren. Die Datenlage zu Biomarkern für die Anwendung der CDK4/6-Inhibitoren wächst, was auch eine Weiterentwicklung für andere molekulare Subtypen bedeuten könnte. Beim HER2+, metastasierten Mammakarzinom wurden neue Substanzen entwickelt, die auch nach massiver Vorbehandlung und/oder zerebraler Metastasierung noch gute Remissionen zeigen. Ebenso wächst das Wissen um zielgerichtete Therapien mit Antikörper-Wirkstoff-Konjugaten (ADC) gegen Trop-2, die bei triple-negativen Mammakarzinomen (TNBC) eine therapeutische Bereicherung bedeuten könnten. Des Weiteren steht das Verständnis des Fertilitätserhaltes nach einer Brustkrebserkrankung im klinischen Fokus. Hier schaffen gepoolte Analysen einen neuen Einblick.

\section{Introduction}

This review summarises the latest data on the treatment of patients with advanced breast cancer and on new biomarkers. It also reports a large pooled analysis of fertility and pregnancy after breast cancer treatment. This type of therapeutic impact on quality of life plays a special role in the ever-improving therapies established in recent years $[1-6]$. This paper considers full publications and presentations at the current major cancer and breast cancer meetings such as ESMO (European Society for Medical Oncology) 2020 and SABCS (San Antonio Breast Cancer Symposium) 2020.

\section{Treatment of Patients with Metastatic Breast Cancer}

\section{Chemotherapy agents partnered with immune checkpoint inhibitors (KEYNOTE-355)}

Publication of the IMpassion131 [7] and IMpassion130 trial [8] raises the question of which chemotherapy agents would best be partnered with anti-PD-1/PD-L1 therapy. More data to clarify this issue are of particular interest here. The IMpassion130 trial compared first-line therapy with nab-paclitaxel and atezolizumab to nab-paclitaxel monotherapy in patients with advanced TNBC. Both progression-free survival and overall survival in PD-L1-positive patients improved [9]. The IMpassion131 trial employed paclitaxel dissolved with Cremophor as a combination agent rather than Nab-paclitaxel and failed to meet its primary endpoint (i.e., improvement in PFS [Progression Free Survival]). Atezolizumab 
thus failed to provide any further benefit in this trial compared with paclitaxel therapy alone [7]. This even led to a warning from the US Food and Drug Administration (FDA) against combining atezolizumab with paclitaxel. However, both studies need to be studied in more detail. In the IMpassion 130 study, almost $40 \%$ of patients did not receive adjuvant or neoadjuvant treatment, i.e., they were treatment-naïve patients. Moreover, no patients were enrolled in the trial who metastasised within 12 months following adjuvant or neoadjuvant treatment. The number of patients with PD-L1-positive tumour in the IMpassion131 trial was relatively low, with 100 patients in each arm. In addition, 30\% of the patients in this study showed de novo metastases, i.e., they were also treatment-naïve, while $70 \%$ of the patients had undergone prior treatment. Since about half of the patients had received taxanes or anthracyclines, the question arises as to what the remaining 20 patients were treated with. This data can only be extracted from the final publications.

Recently, data from the KEYNOTE-355 trial was presented, which was able to provide further insight into this issue. The KEYNOTE-355 trial compared a combination therapy of chemotherapy plus pembrolizumab with chemotherapy alone. Chemotherapy comprised either nab-paclitaxel (31.6\%), paclitaxel (13.5\%) or gemcitabine/carboplatin $(54.9 \%)$, as preferred by the physician. In the overall study, adding pembrolizumab to chemotherapy benefited progression-free survival in the group of patients with a CPS score for PD-L1 of $\geq 10$, with a hazard ratio (HR) of 0.65 (95\% Cl: 0.49-0.86) [10]. In the chemotherapy partner subgroups, the HR was 0.57 (95\% Cl: 0.34-0.95) for nab-paclitaxel, 0.33 (95\% Cl: $0.14-0.76)$ for paxlitaxel and 0.77 (95\% Cl: $0.53-$ 1.11) for gemcitabine/carboplatin [11]. Similar results were seen for patients with a CPS score of $\geq 1$ and in the overall trial population. Thus, KEYNOTE-355 did not demonstrate that the combination partner nab-paclitaxel vs. paclitaxel made a difference in the efficacy of pembrolizumab.

Whether the three trials (IMpassion130/131 and KEYNOTE355) can generally be compared with each other with regard to this question will only become clear once sufficient follow-up time for overall survival is available from all trials. It should be noted that the effect of the immune checkpoint inhibitors was only shown with a treatment-free interval of more than 12 months; in the KEYNOTE-355 trial, no effect was shown with a treatment-free interval of less than 12 months, and the IMpassion130 and -131 trials, too, only enrolled patients who had not undergone treatment for at least 12 months since the end of adjuvant chemotherapy.

\section{Oral taxanes}

A new oral formulation of paclitaxel was reported at SABCS 2019 a year ago [12]. Only small amounts of paclitaxel are absorbed orally because it is removed from the cell by the P-glycoprotein pump (P-gp) [13]. Specific inhibition of P-gp with the agent encequidar increases the absorption of oral paclitaxel. In the phase III KX-ORAX-001 trial, 360 patients were randomised 2:1 to treatment with oral paclitaxel plus encequidar or to treatment with intravenous paclitaxel. Eligible were patients with advanced breast cancer who had not been treated with a taxane within the last year [14]. In both groups, most patients had ER+/HER- breast cancer (56\%/49\%). The primary endpoint of the trial was to demonstrate an improvement in overall response rate with the encequidar combination. In the treatment arm with oral taxane, a response was seen in $40.4 \%$ of cases, whereas in the group with parenteral taxane this was true in only $25.6 \%$ of patients $(p=0.005)$. This difference was statistically significant $(p=0.005)$. A 2019 analysis revealed no difference in PFS with an HR of $0.76(95 \% \mathrm{Cl}: 0.551-1.049)$. However, an HR of 0.684 ( $95 \% \mathrm{Cl}: 0.475-0.985)$ demonstrated a benefit in terms of overall survival for the oral taxane. In terms of side effects, neuropathies $\geq$ grade 2 in particular occurred less frequently with the oral taxane at 7.6 vs. $31.1 \%$ with the intravenous taxane. Alopecia was also observed less frequently. Diarrhoea, nausea and vomiting occurred more frequently [14]. Analyses of this trial conducted in Central and South America with a longer follow-up period have now been presented [14]. The analysis regarding PFS now reached statistical significance (HR: 0.739 ; $95 \%$ Cl: $0.561-$ 0.974). Overall survival also yielded results similar to those of the primary analysis (HR: $0.735 ; 95 \% \mathrm{Cl}: 0.556-0.972$ ). The combination of oral taxane and encequidar is currently (March 2021) under review by the US Food and Drug Administration (FDA).

Another oral taxane is tesetaxel. It is a modified taxane that is not eliminated from the cell by the P-gp and can therefore also be administered orally. The oral bioavailability is $56 \%$. In addition, tesetaxel is markedly more soluble than paclitaxel and docetaxel [15]. Dosed at $27 \mathrm{mg} / \mathrm{m}^{2}$, it is taken once every 3 weeks without premedication. Recently, the outcomes of the phase III CONTESSA trial were reported. This trial had enrolled ER+/HER2- patients who had received no or just one line of chemotherapy in the metastatic setting. In addition, the (neo)adjuvant situation called for treatment with a taxane. Any number of prior endocrine treatments were accepted. Patients were randomised 1:1 to receive treatment with tesetaxel plus capecitabine or capecitabine alone. The primary endpoint of the trial was PFS. Secondary endpoints of the trial included overall survival and overall response rate. Most of the patients enrolled had not received any prior treatment in the metastatic setting (93\%). Thus, the study population comprised mainly patients who received their first treatment in the metastatic setting.

The primary endpoint of the trial was met. With a median follow-up of 13.9 months, the addition of tesetaxel improved median progression-free survival from $6.9(95 \% \mathrm{Cl}: 5.6-8.3)$ to 9.8 (95\% Cl: 8.4-12.0) months. The HR was 0.716 (95\% Cl: $0.573-$ $0.895 ; p=0.003$ ) in favour of tesetaxel plus capecitabine [15]. It is not surprising that combined treatment is superior to monotherapy in terms of response rate and progression-free survival. Whether these promising results become relevant for clinical practice depends on the data on overall survival, which have not been analysed so far because the number of events is too low. Neuropathy grade III-IV developed in $5.9 \%$ of patients treated with tesetaxel [15].

According to a press release, however, further approval of the agent tesetaxel is not being pursued. Reasons were not initially given [16]. 
- Table 1 Impact of molecular subtypes on the effect of ribociclib and the effect on prognosis in the treatment groups regarding progression-free survival.

\begin{tabular}{|c|c|c|c|c|c|c|}
\hline & \multicolumn{2}{|c|}{$\begin{array}{l}\text { Comparison of ribociclib+ER } \\
\text { vs. ET monotherapy* }\end{array}$} & \multicolumn{2}{|c|}{$\begin{array}{l}\text { Comparison of molecular subtype } \\
\text { vs. Luminal A group in patients } \\
\text { treated with ribociclib + endocrine } \\
\text { therapy }\end{array}$} & \multicolumn{2}{|c|}{$\begin{array}{l}\text { Comparison of molecular subtype } \\
\text { vs. Luminal A group in patients } \\
\text { treated with endocrine mono- } \\
\text { therapy }\end{array}$} \\
\hline & Hazard Ratio & p-value & Hazard Ratio & p-value & Hazard Ratio & p-value \\
\hline Luminal A & 0.63 & $<0.001$ & Reference & & Reference & \\
\hline Luminal B & 0.52 & $<0.001$ & 1.17 & 0.35 & 1.68 & 0.00055 \\
\hline HER2 enriched & 0.39 & $<0.001$ & 1.76 & 0.00082 & 1.47 & $<0.0001$ \\
\hline Basal-like & 1.15 & 0.7672 & 5.1 & $<0.0001$ & 3.05 & 0.004 \\
\hline Normal-like & not shown & & 0.98 & 0.93 & 1.69 & 0.0025 \\
\hline
\end{tabular}

\section{Some new substances in the treatment of advanced breast cancer have not been convincing}

Even though significant progress has been made with numerous agents in recent years, there are still prominent examples of trials in which the compounds studied failed to achieve any improvement in efficacy.

One such example is the HDAC (histone deacetylase) inhibitor entinostat.

Following promising data from a randomised phase II trial for patients with advanced HR+/HER2- breast cancer, which demonstrated that both PFS and OS improved with the addition of entinostat to exemestane [17], a phase III E2112 trial was conducted for confirmation. Eligible for enrolment were patients with HR+/HER2 - advanced breast cancer who had progressed on a non-steroidal aromatase inhibitor and who had not received more than one prior chemotherapy. 608 patients received either exemestane plus placebo or exemestane plus entinostat. About one third of the patients had been treated with a CDK4/6 inhibitor before enrolment. The trial was negative. No difference in either PFS or OS was seen between the treatment arms with relatively short median PFS times of 3.3 and 3.1 months. Median overall survival was 23.4 versus 21.7 months.

The other trial, which also followed a positive randomised phase II trial for patients with advanced TNBC, was the IPATunity130 phase III trial [18]. This trial studied the Akt inhibitor ipatasertib. The Akt kinase signalling pathway is one of the main signal transduction pathways from the cell surface to the nucleus. It can be activated by a variety of molecular changes such as PTEN loss, PI3K mutations, or AKT1 mutations. Since approximately $35 \%$ of all TNBC tumours exhibit one of these molecular alterations, targeted therapy may be important for a clinically significant group of patients. The phase II LOTUS trial published previously reported an $\mathrm{HR}$ of 0.60 (95\% Cl: 0.37-0.98) [19] for PFS in the overall population $(\mathrm{N}=93)$. In the group of patients with PTEN/AKT1/PIK3CA alteration $(n=33)$, the HR was $0.44(95 \% \mathrm{Cl}$ : 0.20-0.99) [19]. The IPATunity130 trial now attempted to replicate the outcomes through a randomised phase III trial. It studied 255 patients with advanced TNBC and a PIK3CA/AKT1/PTEN alteration in the first line of therapy, i.e., without prior treatment of the advanced breast cancer. Patients were treated with either paclitaxel weekly $80 \mathrm{mg} / \mathrm{m}^{2}$ or paclitaxel weekly plus $400 \mathrm{mg}$ ipatasertib per day for 21 days followed by a week off. With regard to the primary endpoint PFS at a median follow-up time of 8.3 months, no difference was seen between both treatment arms. Median PFS times were short at 6.1 (95\% Cl: 5.5-9.0) months in the paclitaxel arm and 7.4 months (95\% Cl: 5.6-8.5) in the ipatasertib plus paclitaxel arm (HR: 1.02; $95 \% \mathrm{Cl}: 0.71-$ 1.45).

Both trials emphasise the importance of validating initially good efficacy signals in larger phase III trials.

\section{Biomarker}

\section{PAM50 in treatment with CDK4/6 inhibitors in the metastatic setting (ML2,3,7).}

Combining endocrine treatment with CDK4/6 inhibitors is the standard of care for first line therapy in patients with advanced ER+/HER2- breast cancer. So far, there are no known prognostic or predictive markers, except for hormone receptor status, that could predict a greater or lesser benefit of endocrine monotherapy or combined treatment with CDK4/6 inhibitors plus endocrine therapy. It is known that the histopathological determination of the oestrogen receptor, progesterone receptor, HER2 status, and the grading can roughly classify the patients into intrinsic subtypes. However, in over $20 \%$ of cases, this assessment does not match the molecular subtype [20]. Since CDK4/6 inhibitors have demonstrated their efficacy, particularly in molecular luminal and HER2-amplified cell lines [21], one can reasonably pose this question in clinical populations as well. This has now been undertaken through a pooled analysis of the MONALEESA trials (MONALEESA-2 [22], MONALEESA-3 [23, 24], MONALEESA-7 [25, 26]) [27]. In 1160 of the total population of 2066 patients, it was possible to analyse a tumour sample with the PAM50 classifier with regard to the molecular subtype. Of these 1160 patients, $47 \%$ had subtype luminal A, $24 \%$ had subtype luminal B, $13 \%$ had subtype HER2, $3 \%$ had subtype Basal-like and $14 \%$ had subtype Normallike. The HR for these groups are listed in - Table 1 . The $13 \%$ of 


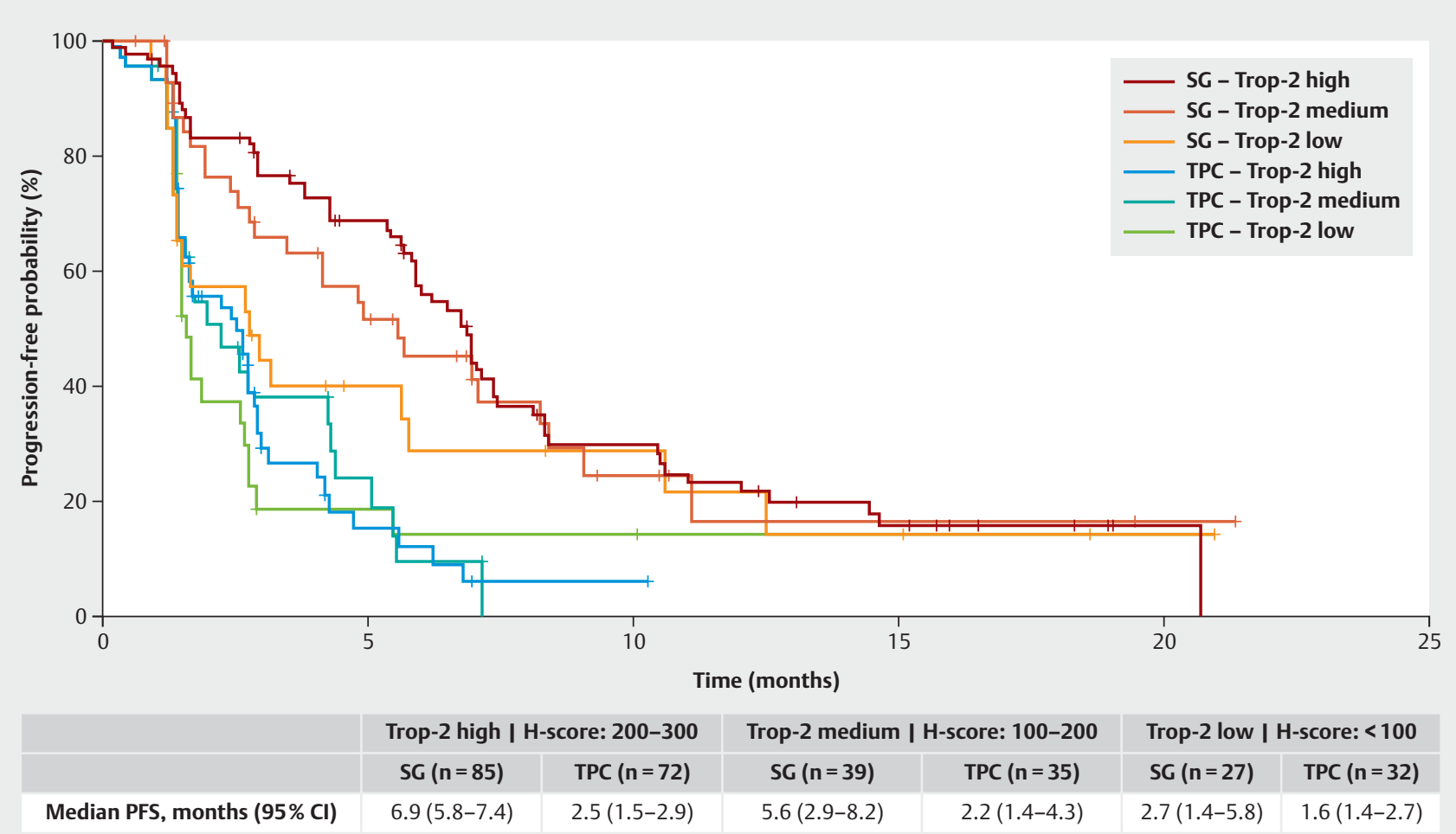

Assessed in brain metastases-negative population. Trop-2 expression determined in archival samples by validated immunohistochemistry assay and $\mathrm{H}$-scoring.

H-score: histochemical score, PFS: progression-free survival, SG: sacituzumab govitecan, TPC: treatment of physician's choice, Trop-2: trophoblast cell surface antigen-2.

Fig. 1 Progression-free survival in the ASCENT trial by Trop-2 expression [31].

patients in the HER2 subgroup benefited most from treatment with ribociclib (HR: 0.39; $<<0.001$ ). The few patients with the Basal-like subtype ( $3 \%$ ) did not benefit from treatment with ribociclib (HR: 1.15; $p=0.7672$ ) [27]. Thus, these results confirm the preclinical data on CDK4/6 inhibitors [21]. Although interesting data was generated by this analysis, it is unlikely that it will affect clinical practice. However, the outcomes could be used to identify suitable patient populations for treatment once CDK4/6 inhibitors are introduced into adjuvant therapy.

\section{Biomarker analysis of the ASCENT trial in HER2-positive metastatic breast cancer}

One class of substances that has significantly enhanced the therapeutic landscape since its approval for advanced HER2-positive breast cancer and for patients without complete response after neoadjuvant anti-HER2 chemotherapy are the so-called antibody drug conjugates (ADCs). A linker binds cytotoxic substances to antibodies. This allows not only target molecules driving the prognosis of cancer to be a treatment target, but also molecules only found on the cell surface as "anchors". This was shown, for example, with the ADC trastuzumab-deruxtecan (T-DXd), which also demonstrated its efficacy in patients with low HER2 expression [28]. In patients with triple-negative breast cancer, the ADC sacituzumab govitecan (SG) represents a new therapy with high efficacy even after some prior therapies $[29,30]$. It binds to the target molecule Trop-2, which has been detected in patients with breast cancer. Compared with chemotherapy as preferred by the physician, sacituzumab govitecan significantly improved both PFS (HR: 0.41) and overall survival (HR: 0.48), as shown in the ASCENT trial [29]. In this context, it is interesting to ask whether the expression levels of Trop-2 might affect the efficacy of the ADC. Hypothetically, any effect on efficacy should not be significant. A biomarker analysis of the ASCENT trial addressed precisely this question. In addition, the TNBC patient population was also tested for BRCA1/2 mutations [31]. Trop-2 expression was determined by immunohistochemistry and patients were classified into high, medium and low Trop-2 expression groups. The analysis included 468 patients altogether. Trop-2 expression was assessed in 290 patients, of whom $54.1 \%$ showed high expression, $25.5 \%$ medium expression and 20.3\% low expression. Of the 292 patients screened for BRCA1/2 mutations, $11.6 \%$ presented with a mutation. In the group of patients who had been treated with chemotherapy, Trop-2 expression did not impact prognosis (median PFS times: 2.5 months [high expression]; 2.2 months [medium expression]; 1.6 months [low expression]). In the sacituzumab govitecan arm, the difference between the medium and high Trop-2 expression groups was also small. In patients with high Trop-2 expression, the median PFS was 6.9 months (95\% Cl: $5.8-7.4)$ and in those with intermediate expression 5.6 months (95\% Cl: 2.9-8.2). However, low expression correlated with a median PFS of only 2.7 months (95\% Cl: 1.4-5.8) [31] (• Fig. 1). Nevertheless, PFS improved significantly in all groups. BRCA1/2 status did not affect 
the efficacy of sacituzumab govitecan [31]. This analysis thus illustrates that principle treatment with an appropriate ADC may also be effective in tumours with lower target molecule expression.

\section{Biomarkers as selection criterion in patients undergoing endocrine therapy}

Many of the (adjuvant) trials on multigene tests or other biomarkers published in recent years employed a trial design investigating whether different characteristics in a patient population resulted in similar prognoses, rather than comparing different treatment regimens, including the endocrine part of the ADAPT trial [32]. The ADAPT trial on HR+/HER- early breast cancer with $0-3$ affected lymph nodes compared the following patients:

A. Patients with an Oncotype DX ${ }^{\circledR}$ Recurrence Score of $0-11$ at initial diagnosis $(n=868)$

B. Patients with an Oncotype DX Recurrence Score of 12-25 at initial diagnosis and a decline in $\mathrm{Ki}-67$ to $\leq 10 \%$ at re-biopsy after 3 weeks of preoperative endocrine treatment $(n=1422)$

Due to the difference in patient selection, both groups differed in most characteristics. The authors emphasised that this was not a comparison of groups in a randomised trial. Both groups had a comparable probability of relapse-free survival. In group $A$, the probability of disease-free survival at 5 years was $93.9 \%(95 \% \mathrm{Cl}$ : 91.8-95.4), whereas in group B it was 92.6\% (95\% Cl: 90.8-94.0). It was ruled out that the difference with a one-tailed $95 \%$ confidence interval was greater than the prespecified 3.3\%. Similar outcomes were obtained for the overall survival analysis, the distant metastasis-free survival analysis, and for patients under the age of 50 years and those over the age of 50 years. The non-inferiority of group $B$, independent of menopausal status, demonstrated that dynamic Ki-67 assessment can close a prognostic gap in premenopausal breast cancer patients with limited lymph node involvement and an RS of 12-25 (see RxPONDER trial).

The only variables of prognostic significance in the group of patients analysed were lymph node status (0-2 malignant lymph nodes vs. 3 lymph nodes), tumour stage (pT2-4 vs. pT0-1) and progesterone receptor expression (ordinal variable with $10 \%$ increments) [32]. Thus, it can be stated that in the heterogeneous group recruited in the ADAPT trial, only lymph node status, tumour size and progesterone receptor expression were independent predictors of prognosis.

\section{CTC as a marker for monitoring treatment in metastatic breast cancer}

One biomarker that has been studied for decades is circulating tumour cells in the blood (CTCs) [33, 34]. In both patients with early breast cancer and those with advanced disease, the presence of CTCs correlates with unfavourable prognosis. One question arising from this observation is whether the presence of CTCs in the course of the disease may be tapped as a monitoring marker for treatment. An international pooled analysis with 4079 patients addressing this issue has now been presented [35]. The paper focused on trials in which CTC measurements were available prior to as well as during the treatment. This international analysis included a total of 14 trials. For the overall population and for the molecular subtypes, groups of patients who continued to have
- Table 2 Overall survival times for patients with and without CTC elimination during observation and the corresponding hazard ratios (from [35]).

\begin{tabular}{|c|c|c|c|}
\hline & & \multicolumn{2}{|c|}{$\begin{array}{l}\text { CTC status at treatment baseline/ } \\
\text { CTC status during the course of } \\
\text { treatment }\end{array}$} \\
\hline & & $\begin{array}{l}\mathrm{CTC}+1 \\
\mathrm{CTC}+\end{array}$ & $\mathrm{CTC}+/ \mathrm{CTC}-$ \\
\hline \multirow[t]{2}{*}{$\begin{array}{l}\text { Overall } \\
\text { population }\end{array}$} & $\begin{array}{l}\text { median } \\
\text { OS }\end{array}$ & 17.87 & 32.2 \\
\hline & HR & 1 & 0.49 (95\% Cl: 0.44- 0.54) \\
\hline \multirow[t]{2}{*}{$\begin{array}{l}\text { HER2- } \\
\text { positive }\end{array}$} & $\begin{array}{l}\text { median } \\
\text { OS }\end{array}$ & 22.8 & 36.83 \\
\hline & HR & 1 & 0.54 (95\% Cl: 0.42-0.69) \\
\hline \multirow[t]{2}{*}{ HR-positive } & $\begin{array}{l}\text { median } \\
\text { OS }\end{array}$ & 20.45 & 36.58 \\
\hline & HR & 1 & 0.47 (95\% Cl: $0.41-0.54)$ \\
\hline \multirow[t]{2}{*}{ TNBC } & $\begin{array}{l}\text { median } \\
\text { OS }\end{array}$ & 9.38 & 20.47 \\
\hline & HR & 1 & 0.41 (95\% Cl: 0.32-0.52) \\
\hline
\end{tabular}

detectable CTCs both at baseline and during follow-up were compared with patients who were also CTC-positive at baseline but for whom CTCs were no longer detected during follow-up. > Table 2 summarises the findings. Both in the overall population and the subgroups (HR-positive, HER2-positive, TNBC), the elimination of CTCs during the treatment roughly doubled median overall survival. This illustrates that CTC levels during the treatment course can provide a very good indication of the response to treatment and its effect on overall survival. Not unlike with neoadjuvant therapy, the question is whether monitoring patients with positive CTCs prior to treatment and an early change in treatment may benefit those patients with persistent CTCs. However, before these ideas can be applied to routine practice, the trial outcomes must be awaited so that this promising technology does not give rise to false expectations or even endanger patients.

\section{Breast Cancer Index and patient selection for extended adjuvant endocrine therapy}

The aTTom trial ("Adjuvant Tamoxifen Treatment Offer More") compared 5 years of adjuvant tamoxifen therapy with 10 years of tamoxifen [36]. A retrospective analysis of this trial involving 1822 of 6956 patients employed a multigene expression test, which had previously been validated as a strong prognostic factor in several trials. The $\mathrm{BCl}$ test comprises two components, the $\mathrm{H} / \mathrm{I}$ ratio (ratio of the gene expression of HOXB13: IL17BR) and the Molecular Grade Index (MGI), which maps tumour proliferation with 5 genes (BUB1B, CENPA, NEK2, RACGAP1, RRM2) [37, 38]. Moreover, different predictions can be made for the prognosis of patients who underwent treatment with tamoxifen for 5 and 10 years, respectively.

It was analysed whether the $\mathrm{H} / \mathrm{I}$ ratio $(\mathrm{BCI}[\mathrm{H} / \mathrm{I}])$ could help to identify patients who would benefit from extending endocrine 
therapy to 10 years. It was shown that the predicted prognosis for patients treated with 5 years of tamoxifen treatment differed significantly from patients treated with 10 years of tamoxifen $(\mathrm{HR}=0.35 ; 95 \% \mathrm{Cl} 0.15-0.86 ; \mathrm{p}=0.014)$. Thus, the $\mathrm{BCl}(\mathrm{H} / \mathrm{I})$ would be the first test capable of identifying patients who would benefit from extended anti-hormone therapy. However, it must be noted that since the aTTom trial had been conducted, far fewer patients have been treated with adjuvant tamoxifen both in the premenopausal and especially in the postmenopausal setting. The data from the MonarchE trial could also mean that in the future adjuvant treatment could include other active substances. The possible significant predictive value of the $\mathrm{BCl}(\mathrm{H} / \mathrm{I})$ would then have to be explored again.

\section{Progress in the treatment of metastatic HER2-positive breast cancer}

Already in 2019 at the San Antonio Breast Cancer Congress (SABCS), trial results were presented for two agents that still show impressive activity after prior treatment of metastatic HER2-positive breast cancer with T-DM1: The HER2-specific tyrosine kinase inhibitor tucatinib and the antibody-drug conjugate tastuzumabderuxtecan (T-DXd).

In a prospective phase II trial, 612 patients with metastatic HER2+ breast cancer and $\geq 2$ prior treatments including T-DM1 were randomised $2: 1$ to capecitabine/trastuzumab/tucatinib versus capecitabine/trastuzumab/placebo. $48 \%$ of the patients had brain metastases [39]. The addition of tucatinib resulted in a significant benefit in both PFS and OS. Of particular note is that in the subgroup of patients with cerebral metastasis, DFS after one year was $24.9 \%$ in the tucantinib group and $0 \%$ in the control group. At the last SABCS in December 2020, E. Hamilton for the HER2CLIMB trial group confirmed the benefits for the combination with tucatinib in terms of DFS and OS, regardless of hormone receptor status and also in patients with cerebral metastases. Adverse reactions such as diarrhoea and hand-foot syndrome were more frequent in the experimental arm. Approval of tucatinib in Germany is expected in the next few weeks and the drug is currently available in Germany through a compassionate use programme.

In the single-arm phase II DestinyBreast01 trial the antibodydrug conjugate trastuzumab-deruxtecan (T-DXd) displayed a good response of $60.9 \%$ and a PFS of 14.8 months in 184 patients with metastatic HER2-positive breast cancer (13\% of whom had stable brain metastases) after prior T-DM1 treatment [28]. Apart from mild to moderate haematological and gastrointestinal side effects, interstitial lung disease (ILD) was observed in $13.6 \%$ of all cases and $\geq 3$ rd degree in 3\%. A 20.4-month follow-up yielded a PFS benefit, which increased to 19.4 months in the last analysis. The rate of pulmonary complications did not rise any further. Thus, ILD does not have a cumulative effect, but it does require interdisciplinary management and good patient education and selection. T-DXd approval in Germany depends on the outcome of the two ongoing phase III trials (DestinyBreast06 trial, NCT0449442, and DestinyBreast03 trial, NCT03529110).
- Table 3 Relative risks of pregnancy after malignant disease [40].

\begin{tabular}{|l|l|l|}
\hline Cancer & Relative risk & $\mathbf{9 5 \%} \mathbf{C l}$ \\
\hline Cervix & 0.33 & $0.31-0.35$ \\
\hline Breast & 0.4 & $0.32-0.49$ \\
\hline Leukaemia & 0.4 & $0.27-0.58$ \\
\hline Kidney & 0.42 & $0.18-0.99$ \\
\hline CNS & 0.52 & $0.39-0.69$ \\
\hline Bone & 0.56 & $0.37-0.86$ \\
\hline Ovary & 0.56 & $0.48-0.65$ \\
\hline Hodgkin lymphoma & 0.62 & $0.47-0.82$ \\
\hline All types & 0.65 & $0.55-0.77$ \\
\hline Liver & 0.65 & $0.19-2.26$ \\
\hline Non-Hodgkin lymphoma & 0.66 & $0.53-0.82$ \\
\hline Colon & 0.7 & $0.41-1.17$ \\
\hline Thyroid & 0.82 & $0.65-1.03$ \\
\hline Skin & 0.97 & $0.87-1.09$ \\
\hline bold = estimates for breast cancer & & \\
\hline
\end{tabular}

\section{Treatment Management and Patient Concerns}

\section{Fertility following breast cancer}

Women who develop breast cancer at a young age often face the question of pregnancy after the disease with all its treatments (chemotherapy, radiotherapy, targeted therapy, and endocrine therapy). An international working group has conducted a systematic review with a meta-analysis. They were able to identify a total of 39 studies that could be analysed for the chance of pregnancy, pregnancy complications and maternal mortality.

48513 breast cancer patients were compared with 3289113 healthy women regarding the probability of pregnancy following breast cancer. The relative risk of pregnancy was 0.40 (95\% Cl: 0.32-0.49). In other words, women who had developed breast cancer were $60 \%$ less likely to become pregnant than women in the healthy comparison population. The comparison with other types of cancer, shown in > Table 3 [40], was interesting. Here it is noteworthy that only patients with cervical cancer had a smaller chance of pregnancy after malignant disease than patients with breast cancer. All other tumour entities had a better chance of pregnancy. With regard to the rate of pregnancy complications, a comparison of 3240 breast cancer patients and more than 4800000 women from healthy controls revealed that the risk of caesarean section was increased with a relative risk of $1.14(95 \%$ Cl: 1.04-1.25), of low birth weight (RR: 1.50; $95 \% \mathrm{Cl}: 1.31-1.73$ ) and of preterm birth (RR: 1.45; $95 \% \mathrm{Cl}: 1.11-1.88$ ). However, the risk of congenital anomalies had an RR of 1.68 (95\% Cl: $0.89-$ 2.98). Due to the small number of cases, though, this risk was probably not statistically significant $(p=0.112)$. Further subgroup analyses revealed that the risk of low birth weight was higher in the group of patients who had received chemotherapy for breast 
cancer (RR: 1.662; 95\% Cl: 1.08-2.42) versus patients who did not undergo chemotherapy (RR: 1.05; 95\% Cl: 0.77-1.43) [40].

In terms of relapse-free survival/overall survival (OS) after breast cancer, 2003 (DFS)/3261 (OS) breast cancer patients with a pregnancy post disease were compared with approximately 38000 (PFS)/52000 (OS) patients without pregnancy. In general, pregnancy had protective effects on survival. The HR for DFS was 0.73 (95\% Cl: $0.56-0.94)$ and $0.56(0.46-0.67)$ for overall survival. Moreover, these effects were largely independent of other disease parameters such as nodal status and chemotherapy.

\section{Outlook}

For some treatments with demonstrated definite efficacy, such as the CDK4/6 inhibitors, the next stage will be to establish and understand any sequences with other treatment regimens, as well as to possibly refine the treatment for other molecular subtypes or treatment settings. With the ADC sacituzumab govitecan, another compound from this drug class seems to be gaining a foothold. Tucatinib and trastuzumab-deruxtecan broaden the therapeutic spectrum in metastatic HER2-positive breast cancer.

The insights into biomarkers are broadening our understanding of the mode of action and the potential applications of ADCs in breast cancer. Some expectations regarding Akt kinase inhibitors and HDAC inhibitors could not be confirmed in phase III trials. Nevertheless, the development of new biomarkers and treatment regimens for patients with advanced breast cancer is progressing rapidly, and the therapeutic options are expected to evolve.

\section{Acknowledgements}

This paper was produced in part as a result of funding from the following companies: onkowissen.de; Hexal; Pfizer; Lilly; and Novartis; as well as the PRAEGNANT network, which is supported by Pfizer; Hexal; MSD; Daiichi-Sankyo; Merrimack; Eisai; AstraZeneca; and Novartis. No company had any part in the preparation of this manuscript. Sole responsibility for the content of the manuscript rests with the authors.

\section{Conflict of Interest}

B. A. received honoria and travel grants from AstraZeneca, Genomic Health, Roche, Novartis, Celgene, Lilly, MSD, Eisai, Teva, Tesaro, DaiichiSankyo and Pfizer.

E. B. received honoraria from Novartis, Hexal, BMS, Lilly, Pfizer, Roche, MSD, Bayer, Ipsen, Bluebird, BBraun and onkowissen.de for consulting, clinical research management or medical education activities.

N.D. has received honoraria from MSD, Roche, AstraZeneca, Teva, Mentor, and $\mathrm{MCl}$ Healthcare.

P. A. F. received honoraria from Novartis, Pfizer, Roche, Amgen, Celgene, onkowissen.de, Daiichi-Sankyo, AstraZeneca, Merck-Sharp \& Dohme, Eisai, Puma and Teva. His institution conducts research with funding from Novartis and Biontech.

T. N. F. has participated on advisory boards for Amgen, Daiichi Sankyo, Novartis, Pfizer, and Roche and has received honoraria for lectures from Amgen, Celgene, Daiichi Sankyo, Roche, Novartis and Pfizer.

A.D.H. received speaker and consultancy honoraria from AstraZeneca, Genomic Health, Roche, Novartis, Celgene, Lilly, MSD, Eisai, Teva, Tesaro, Daiichi-Sankyo, Hexal and Pfizer.

C. J. reports personal fees from AstraZeneca, Exact Sciences, Lilly, Novartis and Roche during the conduct of the study.
C. K.-L. has received honoraria from Roche, AstraZeneca, Celgene, Novartis, Pfizer, Lilly, Hexal, Amgen, Eisai, and SonoScape, honoraria for consultancy from Phaon Scientific, Novartis, Pfizer, and Celgene, research funding from Roche, Novartis, and Pfizer, and travel grants from Novartis and Roche.

H.-C. K. has received honoraria from Pfizer, Novartis, Roche, Genomic Health/Exact Sciences, Amgen, AstraZeneca, Riemser, Carl Zeiss Meditec, Teva, Theraclion, Janssen-Cilag, GSK, LIV Pharma, Lily, SurgVision, Onkowissen and MSD, travel support from Carl Zeiss Meditec, LIV Pharma, Novartis, Amgen, Pfizer, Daiichi Sankyo, Tesaro and owns stock of Theraclion SA and Phaon Scientific GmbH.

D. L. received honoraria from Amgen, AstraZeneca, Celgene, Lilly, Loreal, MSD, Novartis, Pfizer, Tesaro, Teva.

M.P. L. has participated on advisory boards for AstraZeneca, Lilly, MSD, Novartis, Pfizer, Eisai, Exact Sciences, Pierre Fabre, Grünenthal, Hexal and Roche and has received honoraria for lectures from MSD, Lilly, Roche, Novartis, Pfizer, Exact Sciences, AstraZeneca, and Eisai. He is editorial board member of medactuell from medac.

V. M. received speaker honoraria from Amgen, Astra Zeneca, DaiichiSankyo, Eisai, Pfizer, MSD, Novartis, Roche, Teva, Seattle Genetics and consultancy honoraria from Genomic Health, Hexal, Roche, Pierre Fabre, Amgen, ClinSol, Novartis, MSD, Daiichi-Sankyo, Eisai, Lilly, Tesaro, Seattle Genetics and Nektar. Institutional research support from Novartis, Roche, Seattle Genetics, Genentech. Travel grants: Roche, Pfizer, Daiichi Sankyo.

E. S. received honoraria from Roche, Celgene, AstraZeneca, Novartis, Pfizer, Tesaro, Aurikamed GmbH, MCI Deutschland GmbH, bsh medical communications GmbH, Onkowissen TV.

M.S. has previously received honoraria for advisory board duty from Roche, Pfizer, Novartis, Astra-Zeneca and Eisai.

A.S. received honoraria from Roche, Celgene, AstraZeneca, Novartis, Pfizer, Zuckschwerdt Verlag GmbH, Georg Thieme Verlag, Aurikamed $\mathrm{GmbH}, \mathrm{MCl}$ Deutschland $\mathrm{GmbH}$, bsh medical communications $\mathrm{GmbH}$ and promedicis $\mathrm{GmbH}$.

F. S. participated on advisory boards for Novartis, Lilly, Amgen and Roche and received honoraria for lectures from Roche, AstraZeneca, MSD, Novartis and Pfizer.

H. T. received honoraria from Novartis, Roche, Celgene, Teva, Pfizer, AstraZeneca and travel support from Roche, Celgene and Pfizer.

C. T. Advisory boards, lectures: Amgen, AstraZeneca, Celgen, DaiichiSankyo, Eisai, Lilly, MSD, Mundipharma, Medapharm, Novartis, Pfizer, Pierre-Fabre, Roche, Tesaro, and Vifor.

M. T. has participated on advisory boards for AstraZeneca, Clovis, Eisai, GSK, Lilly, MSD, Novartis, Pfizer, Exact Sciences, Pierre-Fabre and Roche and has received honoraria for lectures from Clovis, Daiichi Sankyo, GSK, Lilly, MSD, Roche, Novartis, Pfizer, Exact Sciences, and AstraZeneca and has received trial funding by Exact.

C. U. received honoraria from Roche, Novartis, Pfizer, Astra-Zeneca, MSD and onkowissen.de.

M.U. all honoraria went to the institution/employer: Abbvie, Amgen, Astra Zeneca, Celgene, Daichi Sankyo, Eisai, Lilly, MSD Merck, Mundipharma, Myriad Genetics, Pfizer, PUMA Biotechnology, Roche, Sanofi Aventis, Novartis, Pierre Fabre.

M. W. has participated on advisory boards for AstraZeneca, Lilly, MSD, Novartis, Pfizer and Roche.

A.W. participated on advisory boards for Novartis, Lilly, Amgen, Pfizer, Roche, Tesaro, Eisai and received honoraria for lectures from Novartis, Pfizer, Aurikamed, Roche, Celgene.

R.W. has received personal fees/travel support from Agendia, Amgen, Aristo, Astra Zeneca, Boeringer Ingelheim, Carl Zeiss, Celgene, Clinsol, Daiichi-Sankyo, Eisai, Exact Sciences, Genomic Health, Glaxo Smith Kline, Hexal, Lilly, Medstrom Medical, MSD, Mundipharma, Nanostring, Novartis, Odonate, Paxman, Palleos, Pfizer, Pierre Fabre, Puma Biotechnology, Riemser, Roche, Sandoz/Hexal, Seattle Genetics, Tesaro Bio, Teva, Veracyte and Viatris.

The other authors have no conflict of interest to declare for this specific work. 


\section{References}

[1] Ditsch N, Untch M, Thill M et al. AGO Recommendations for the Diagnosis and Treatment of Patients with Early Breast Cancer: Update 2019. Breast Care (Basel) 2019; 14: 224-245

[2] Ditsch N, Untch M, Kolberg-Liedtke C et al. AGO Recommendations for the Diagnosis and Treatment of Patients with Locally Advanced and Metastatic Breast Cancer: Update 2020. Breast Care (Basel) 2020; 15: 294-309

[3] Tesch H, Müller V, Wöckel A et al. Update Breast Cancer 2020 Part 4 Advanced Breast Cancer. Geburtshilfe Frauenheilkd 2020; 80: 11151122

[4] Schneeweiss A, Hartkopf AD, Müller V et al. Update Breast Cancer 2020 Part 1 - Early Breast Cancer: Consolidation of Knowledge About Known Therapies. Geburtshilfe Frauenheilkd 2020; 80: 277-287

[5] Lüftner D, Schneeweiss A, Hartkopf AD et al. Update Breast Cancer 2020 Part 2 - Advanced Breast Cancer: New Treatments and Implementation of Therapies with Companion Diagnostics. Geburtshilfe Frauenheilkd 2020; 80: 391-398

[6] Huober ], Schneeweiss A, Hartkopf AD et al. Update Breast Cancer 2020 Part 3 - Early Breast Cancer. Geburtshilfe Frauenheilkd 2020; 80: 11051114

[7] Miles DW, Gligorov J, André F et al. Primary results from IMpassion131, a double-blind placebo-controlled randomised phase III trial of first-line paclitaxel (PAC) \pm atezolizumab (atezo) for unresectable locally advanced/metastatic triple-negative breast cancer (mTNBC). Ann Oncol 2020; 31 (Suppl. 4): S1142-S1215. doi:10.1016/annonc/annonc325

[8] Schmid P, Adams S, Rugo HS et al. IMpassion130: updated overall survival (OS) from a global, randomized, double-blind, placebo-controlled, Phase III study of atezolizumab (atezo) + nab-paclitaxel (nP) in previously untreated locally advanced or metastatic triple-negative breast cancer (mTNBC). J Clin Oncol 2019; 37 (15 Suppl.): 1003-1003

[9] Schmid P, Adams S, Rugo HS et al. Atezolizumab and Nab-Paclitaxel in Advanced Triple-Negative Breast Cancer. N Engl J Med 2018; 379: 2108-2121

[10] Cortes ], Cescon DW, Rugo HS et al. KEYNOTE-355: Randomized, double-blind, phase III study of pembrolizumab + chemotherapy versus placebo + chemotherapy for previously untreated locally recurrent inoperable or metastatic triple-negative breast cancer. J Clin Oncol 2020; 38 (15 Suppl.): 1000

[11] Rugo HS, Schmid P, Cescon DW et al. Additional efficacy endpoints from the phase 3 KEYNOTE-355 study of pembrolizumab plus chemotherapy vs. placebo plus chemotherapy as first-line therapy for locally recurrent inoperable or metastatic triple-negative breast cancer. San Antonio Breast Cancer Symposium 2020; 2020: GS3-01. Accessed March 16, 2021 at: https://www.abstractsonline.com/pp8/\#!/9223/presentation/ 1725

[12] Umanzor G, Cutler DL, Barrios FJ et al. Oral paclitaxel with encequidar: The first orally administered paclitaxel shown to be superior to IV paclitaxel on confirmed response and survival with less neuropathy: A phase III clinical study in metastatic breast cancer. Cancer Res 2020; 80 (Suppl. 4): GS6-01

[13] Jang SH, Wientjes MG, Au JL. Kinetics of P-glycoprotein-mediated efflux of paclitaxel. J Pharmacol Exp Ther 2001; 298: 1236-1242

[14] Umanzor G, Rugo HS, Barrios F] et al. Oral Paclitaxel and Encequidar (oPac+E) versus IV paclitaxel (IVPac) in the Treatment of Metastatic Breast Cancer (mBC) Patients (Study KX-ORAX-001): Progression Free Survival (PFS) and Overall Survival (OS) Updates. San Antonio Breast Cancer Symposium 2020; 2020: PD1-08(4). Accessed March 16, 2021 at: https://www.abstractsonline.com/pp8/\#!/9223/presentation/716
[15] O'Shaughnessy J, Schwartzberg L, Piccart M et al. Results from CONTESSA: A phase 3 study of tesetaxel plus a reduced dose of capecitabine versus capecitabine alone in patients with HER2-, hormone receptor + $(\mathrm{HR}+)$ metastatic breast cancer (MBC) who have previously received a taxane. San Antonio Breast Cancer Symposium 2020; 2020: GS4-01. Accessed March 16, 2021 at: https://www.abstractsonline.com/pp8/ \#!/9223/session/155

[16] businesswire. Odonate Therapeutics Announces Discontinuation of Development of Tesetaxel. Accessed March 23, 2021 at: https://www. businesswire.com/news/home/20210322005211/en/OdonateTherapeutics-Announces-Discontinuation-of-Development-of-Tesetaxel

[17] Yardley DA, Ismail-Khan RR, Melichar B et al. Randomized phase II, double-blind, placebo-controlled study of exemestane with or without entinostat in postmenopausal women with locally recurrent or metastatic estrogen receptor-positive breast cancer progressing on treatment with a nonsteroidal aromatase inhibitor. J Clin Oncol 2013; 31: 2128-2135

[18] Dent R, Kim S-B, Oliveira M et al. Double-blind placebo (PBO)-controlled randomized phase III trial evaluating first-line ipatasertib (IPAT) combined with paclitaxel (PAC) for PIK3CA/AKT1/PTEN-altered locally advanced unresectable or metastatic triple-negative breast cancer (aTNBC): primary results from IPATunity 130 Cohort A. San Antonio Breast Cancer Symposium 2020; 2020: GS3-04. Accessed March 16, 2021 at: https://www.abstractsonline.com/pp8/\#!/9223/presentation/ 672

[19] Kim SB, Dent R, Im SA et al. Ipatasertib plus paclitaxel versus placebo plus paclitaxel as first-line therapy for metastatic triple-negative breast cancer (LOTUS): a multicentre, randomised, double-blind, placebo-controlled, phase 2 trial. Lancet Oncol 2017; 18: 1360-1372

[20] Sotiriou C, Pusztai L. Gene-expression signatures in breast cancer. N Eng J Med 2009; 360: 790-800

[21] Finn RS, Dering J, Conklin D et al. PD 0332991, a selective cyclin D kinase 4/6 inhibitor, preferentially inhibits proliferation of luminal estrogen receptor-positive human breast cancer cell lines in vitro. Breast Cancer Res 2009; 11: R77

[22] Hortobagyi GN. Ribociclib for HR-Positive, Advanced Breast Cancer. N Engl J Med 2017; 376: 289

[23] Slamon DJ, Neven P, Chia S et al. Phase III Randomized Study of Ribociclib and Fulvestrant in Hormone Receptor-Positive, Human Epidermal Growth Factor Receptor 2-Negative Advanced Breast Cancer: MONALEESA-3. J Clin Oncol 2018; 36: 2465-2472

[24] Slamon D], Neven P, Chia S et al. Overall Survival with Ribociclib plus Fulvestrant in Advanced Breast Cancer. N Engl J Med 2020; 382: 514-524

[25] Tripathy D, Im SA, Colleoni M et al. Ribociclib plus endocrine therapy for premenopausal women with hormone-receptor-positive, advanced breast cancer (MONALEESA-7): a randomised phase 3 trial. Lancet Oncol 2018; 19: 904-915

[26] Im SA, Lu YS, Bardia A et al. Overall Survival with Ribociclib plus Endocrine Therapy in Breast Cancer. N Engl J Med 2019; 381: 307-316

[27] Prat A, Chaudhury A, Solovieff $\mathrm{N}$ et al. Correlative biomarker analysis of intrinsic subtypes and efficacy across the MONALEESA Phase III studies. San Antonio Breast Cancer Symposium 2020; 2020: GS1-04. Accessed March 16, 2021 at: https://www.abstractsonline.com/pp8/\#!/9223/ presentation/665

[28] Modi S, Park H, Murthy RK et al. Antitumor Activity and Safety of Trastuzumab Deruxtecan in Patients With HER2-Low-Expressing Advanced Breast Cancer: Results From a Phase Ib Study. J Clin Oncol 2020; 38: 1887-1896

[29] Bardia A, Tolaney SM, Loirat D et al. ASCENT: A randomized phase II study of sacituzumab govitecan (SG) vs. treatment of physician's choice (TPC) in patients (pts) with previously treated metastatic triple-negative breast cancer (mTNBC). Ann Oncol 2020; 31 (Suppl. 4): S1142-S1215. doi:10.1016/annonc/annonc325 
[30] Bardia A, Mayer IA, Vahdat LT et al. Sacituzumab Govitecan-hziy in Refractory Metastatic Triple-Negative Breast Cancer. N Engl J Med 2019; 380: 741-751

[31] Hurvitz SA, Tolaney SM, Punie K et al. Biomarker evaluation in the phase 3 ASCENT study of sacituzumab govitecan versus chemotherapy in patients with metastatic triple-negative breast cancer San Antonio Breast Cancer Symposium 2020; 2020: GS3-06. Accessed March 16, 2021 at: https://www.abstractsonline.com/pp8/\#!/9223/presentation/674

[32] Harbeck N, Johnston S, Fasching P et al. High Ki-67 as a biomarker for identifying patients with high risk early breast cancer treated in monarchE. San Antonio Breast Cancer Symposium 2020; 2020: PD2-01. Accessed March 16, 2021 at: https://www.abstractsonline.com/pp8/ \#!/9223/presentation/781

[33] Braun S, Marth C. Circulating tumor cells in metastatic breast cancer toward individualized treatment? N Engl J Med 2004; 351: 824-826

[34] Cristofanilli M, Budd GT, Ellis M] et al. Circulating tumor cells, disease progression, and survival in metastatic breast cancer. $\mathrm{N}$ Engl J Med 2004; 351: 781-791
[35] Janni W], Yab TC, Hayes DF et al. Clinical utility of repeated circulating tumor cell (CTC) enumeration as early treatment monitoring tool in metastatic breast cancer (MBC) - a global pooled analysis with individual patient data. San Antonio Breast Cancer Symposium 2020; 2020: GS408. Accessed March 16, 2021 at: https://www.abstractsonline.com/ pp8/\#!/9223/presentation/685

[36] Gray RG, Rea D, Handley K et al. aTTom: Long-term effects of continuing adjuvant tamoxifen to 10 years versus stopping at 5 years in 6,953 women with early breast cancer. J Clin Oncol 2013; 31 (18 Suppl.): 5

[37] Ma XJ, Salunga R, Dahiya $S$ et al. A five-gene molecular grade index and HOXB13:IL17BR are complementary prognostic factors in early stage breast cancer. Clin Cancer Res 2008; 14: 2601-2608

[38] Bartlett J, Sgroi D, Treuner K et al. Trans-aTTom: Breast Cancer Index for prediction of endocrine benefit and late distant recurrence (DR) in patients with $\mathrm{HR}+$ breast cancer treated in the adjuvant tamoxifen - To offer more? (aTTom) trial. J Clin Oncol 2019; 37 (15 Suppl.): 505

[39] Murthy RK, Loi S, Okines A et al. Tucatinib, Trastuzumab, and Capecitabine for HER2-Positive Metastatic Breast Cancer. N Engl J Med 2020; 382: 597-609

[40] Blondeaux E, Perachino M, Bruzzone M et al. Chances of pregnancy after breast cancer, reproductive and disease outcomes: a systematic review and meta-analysis San Antonio Breast Cancer Symposium 2020; 2020: GS3-09. Accessed March 16, 2021 at: https://www.abstractsonline. com/pp8/\#!/9223/presentation/677 